Results Case 1: A 28 year old HIV-positive man presented 96 hours after taking a deliberate overdose of $40 \times 400 \mathrm{mg}$ raltegravir tablets. He developed mild symptoms of diarrhoea, abdominal cramps and a sore chest. Results post-overdose: electrolytes and renal function: normal; liver function tests: ALT 58, others normal; creatinine kinase 67; haematology: normal; therapeutic drug monitoring (TDM) results: raltegravir not detected 96 hours post overdose. There were no serious complications. Case 2: A 52 year old HIV-positive man presented 24 hours after taking a deliberate overdose of $18 \times$ Truvada (tenofovir disaproxil fumarate/emtricitabine). He had no symptoms related to the overdose. Results post-overdose: urinalysis: normal; electrolytes and renal: phosphate 0.75, creatinine 94; TDM: 24 hours: 207 ng/ml; 48 hours: $80 \mathrm{ng} / \mathrm{ml}$; 72 hours: $16 \mathrm{ng} / \mathrm{ml}$; 192 hours: 2 $\mathrm{ng} / \mathrm{ml}$. There were no serious complications.

Discussion/conclusion The patients in our case series showed few side effects and no serious sequelae as a result of their overdose. There seems to be little guidance available to guide management of such cases.

\section{P059 A REVIEW OF SEXUALLY ACQUIRED PROCTITIS: AN ARRAY OF SYMPTOMS, INVESTIGATIONS AND TREATMENTS}

Elizabeth Nicol, Jonathan Shaw*, Christopher Ward. The Hathersage Integrated Contraception, Sexual Health and HIV Service, Manchester, UK

10.1136/sextrans-2016-052718.113

Introduction With recent lymphogranuloma veneruem (LGV) and Shigella outbreaks amongst men-who-have-sex-with-men (MSM), proctitis has become a prominent clinical issue. There is no UK guideline regarding proctitis management but guidance is available from IUSTI and CDC.

Objectives To review our proctitis cases and generate a clinic policy to standardise practice.

Methods Casenotes coded C4NR between 01/01/14-31/12/15 were reviewed with data collated and analysed via Microsoft Excel.

Results 100 care episodes were reviewed (92 patients, 6 attended twice and 1 thrice). All patients were male; 83 homosexual, 8 bisexual and 1 heterosexual. 67 patients were White British, 31 were HIV positive. Median age was 29 years (range $=18-62$ ). Presenting symptoms were varied with rectal pain (58), discharge (54), and bleeding (44) most common. Proctoscopy in 82 cases found varying signs (32 discharge, 24 oedema, 25 contact bleeding, 10 ulceration). Microscopy was diagnostic of proctitis in 39/ 84 (46.4\%) patients. Physician-requested investigations were:

\begin{tabular}{lll} 
Abstract P059 Table 1 & Sexually acquired proctitis \\
\hline Test & Performed & Positive results \\
\hline Chlamydia trachomatis TMA & 100 & 14 (including 6 LGV) \\
Neisseria gonorrhoea TMA & 100 & 28 \\
Gonococcal culture & 98 & 24 \\
Herpes Simplex PCR & 30 & 9 \\
Treponema pallidum PCR & 27 & 1 \\
Treponemal serology & 93 & 5 \\
Stool culture & 13 & 4 \\
\hline
\end{tabular}

Treatment at initial visit was predominantly doxycycline-based (99/100), with course length varying from 7-21 days.
Concurrent therapies were influenced by clinical findings and reported contacts; predominantly ceftriaxone (53), azithromycin (31), and aciclovir (19).

Conclusions Gonorrhoea incidence was high (28\%), as was herpes when requested (30\%). Updated clinic policy for all proctitis patients includes requesting Herpes Simplex PCR and presumptive treatment for gonorrhoea.

\section{P060 ARE WE 'SPOTTING THE SIGNS?'}

Arnold Fernandes*, Mary-Anne Bailey, Ameeka Thompson, Kate Horn. Royal United Hospital, Bath, UK

\subsection{6/sextrans-2016-052718.114}

Background/introduction In 2014, BASHH/Brook piloted a proforma for identifying risks of child sexual exploitation in sexual health settings in the light of cases of child sexual exploitation identified nationally. Use of the pro-forma was promoted. The form was introduced in our unit in October 2014 following discussion. This replaced the 'under-16's risk-assessment,' used previously.

Aim(s)/objectives To assess as to whether the pro-forma was being used overall, with in-depth analysis of key components of the document.

Methods Retrospective note audit between 01/04/15-30/09/215 conducted. 44 attendees under 18, identified (17 male, 27 female)

Results The form was used in 39/44 (88.6\%). Assessment of Fraser competence was documented in 34/42 (81\%); 2 attendees were over 16 . There was documentation that 'confidentiality clause' was discussed in $37 / 44$ (84.1\%). Age of partner was documented in 35/42 (83.3\%); 2 patients had never had sex and hence were excluded. Name of social worker was documented in $7 / 13(53.8 \%)$ attendees who had indicated they had one. 31 attendees had no social worker. Professional analysis was completed in 16/44 (36.4\%). However, a further 10 notes had comments documented, which increased completion rate to $59.09 \%$. Discussion/conclusion After initial concerns raised by Staff about time taken to complete the form and the qualitative nature of information included in the form, this was incorporated into most consultations including under-18 attendees. Documentation on most key aspects of the pro-forma was generally good, with room for improvement. A feed-back session for staff combined with a further dedicated teaching session on safe-guarding is organised to improve this.

\section{P061 SAFETY OF SINGLE DOSE GENTAMICIN COMPARED WITH MULTIPLE DOSE REGIMENS}

${ }^{1}$ Rachel Hayward*, ${ }^{1}$ Jan Harding, ${ }^{1}$ Robert Molloy, ${ }^{3}$ Lucy Land, ${ }^{1}$ Kate Longcroft-Neal, ${ }^{2}$ Dave Moore, 'Jonathan Ross. ${ }^{1}$ Whittall Street Clinic, Birmingham, UK; ${ }^{2}$ University of Birmingham, Birmingham, UK; ${ }^{3}$ Birmingham City University, Birmingham, UK

\subsection{6/sextrans-2016-052718.115}

Background Traditionally, gentamicin is given eight hourly, guided by drug levels. Several studies have shown that singledaily dosing of gentamicin offers an equal, if not improved, toxicity profile compared to traditional dosing. Single one-off dose gentamicin has been suggested as treatment for gonorrhoea, but its safety has not been reviewed.

Aim Systematically review the frequency and type of adverse events associated with a single dose of intravenous or 